\title{
Levobupivacaine Hydrochloride
}

National Cancer Institute

\section{Source}

National Cancer Institute. Levobupivacaine Hydrochloride. NCI Thesaurus. Code C61805.

The hydrochloride salt of levobupivacaine, an amide derivative with anesthetic property. Levobupivacaine reversibly binds voltage-gated sodium channels to modulate ionic flux and prevent the initiation and transmission of nerve impulses (stabilizing neuronal membrane), thereby resulting in analgesia and anesthesia. In comparison with racemic bupivacaine, levobupivacaine is associated with less vasodilation and has a longer duration of action. 